Voix et Images

volxetimages

\title{
Charles Guérin et la fiction au XIX ${ }^{\mathrm{e}}$ siècle
}

Jean-Pierre Duquette

Volume 1, numéro 2, décembre 1975

Raoul Duguay

URI : https://id.erudit.org/iderudit/290074ar

DOI : https://doi.org/10.7202/290074ar

Aller au sommaire du numéro

Éditeur(s)

Les Presses de l'Université du Québec

\section{ISSN}

0318-9201 (imprimé)

1705-933X (numérique)

Découvrir la revue

Citer cet article

Duquette, J.-P. (1975). Charles Guérin et la fiction au XIX ${ }^{\mathrm{e}}$ siècle. Voix et Images, 1(2), 182-195. https://doi.org/10.7202/290074ar d'utilisation que vous pouvez consulter en ligne.

https://apropos.erudit.org/fr/usagers/politique-dutilisation/ 


\section{Charles Guérin et la fiction au XIX ${ }^{\mathrm{e}}$ siècle}

“Notre troisième roman ${ }^{1}$ ", comme l'a désigné Séraphin Marion. Après l'Influence d'un livre, à mi-chemin entre le récit légendaire et la magie noire, et les Fiancés de 1812, mélodrame pavé de bons sentiments, l'œuvre de Chauveau est bien le troisième texte de la littérature romanesque québécoise. Avec, il est vrai, la Terre paternelle de Patrice Lacombe, parue en feuilleton au même moment.

Deux caractéristiques rapprochent du reste la plupart de ces premiers romans. D'abord l'idée, chez les auteurs eux-mêmes, ou leurs préfaciers, qu'ils n'écrivent pas vraiment un texte romanesque; et, immédiatement, leur refus de voir identifier leurs œuvres aux romans européens du temps-entendons ici les romans français, "ces drames terribles et pantelans [sic], comme Eugène Sue et Frédéric Soulié en ont écrits [re-sic] ».

L'espèce d'auto-censure que s'imposent les romanciers s'explique aisément si l'on songe aux foudres répandues du haut de la chaire, dans des lettres pastorales et autres mandements d'évêques, qui tonnent à qui mieux mieux contre ces histoires pleines "d'immondices et de blasphèmes" que sont les romans et les feuilletons français d'alors. Les "moralistes" emboîtent le pas: Étienne Parent, l'éditeur de notre roman G. H. Cherrier, les romanciers Patrice Lacombe et Antoine Gérin-Lajoie, Edmond Rousseau et tant d'autres. Jusqu'au père Aubert de Gaspé, dont les Anciens Canadiens comportent pourtant bien peu de romanesque, qui affirme ne pas "composer un ouvrage secundum artem", conseillant au lecteur de “jeter promptement ce malheureux livre». Non seulement refuse-t-il le fait même d'écrire, mais plus: il ne veut pas être lu. Presque tous les romanciers de l'époque pourraient reprendre à leur compte cette phrase étonnante de Gérin-Lajoje: "Ce n'est pas un roman que j'écris... " À la limite, on

1. On se réfère toujours à l'édition originale, parue à Montréal chez G.H. Cherrier, en 1853. Une réédition du roman a paru chez M.A. Guérin, éditeur à Montréal en 1973. On a alors simplement reproduit l'édition de la Compagnie de publication de la Revue canadienne (1900). Et l'auteur de l'introduction ne donne aucune précision sur les divers états du texte. II "effleure" tout bonnement "quelques notions et aperçus", en appliquant telle quelle la grille des trente et une fonctions de Propp au récit de Chauveau. 
pourrait comprendre: je fais un roman qui n'en est pas un, j'écris tout en n'écrivant pas, parce qu'on ne doit pas écrire de romans ${ }^{2}$.

Un autre sérieux handicap découle de la vision qu'on a du romancier à ce moment-là. La littérature est considérée comme un simple passetemps, une agréable façon de ne rien faire. Le vieil Aubert de Gaspé le dit lui-même: "J'écris pour m'amuser", ce qui, après tout et vu son âge, est peut-être la vérité. G. H. Cherrier, dans son avis de 1853 , indique bien comment nos premiers hommes de lettres ont souvent été forcés de s'adonner à " mille autres occupations plus profitables, en ce qu'elles rapportaient plus d'argent et même beaucoup plus de considération". Si on veut l'en croire, les choses ont bien changé (depuis 1846?) et le "discrédit» qui frappait les écrivains, comme "le mépris des hommes affairés" à leur endroit dimınuent chaque année. Précisément, on se souvient que les tranches de Charles Guérin parues en feuilleton ${ }^{3}$ en 1846 et 1847 étaient anonymes, et que «forcé, par des occupations plus sérieuses, d'interrompre son travail», Chauveau ne donna à publier que la première partie et la moitié de la seconde seulement. En outre, six ans plus tard, quand l'éditeur Cherrier commence la publication du roman, il avoue que le manuscrit entier se trouvait entre ses mains depuis près d'un an, mais que "des circonstances imprévues [lui] ont fait retarder la publication jusqu'à ce jour».

Quelles sont donc ces "occupations plus sérieuses"? Et comment expliquer le long délai entre le début de la première parution incomplète et l'achat du manuscrit par Cherrier, puis ces "circonstances imprévues" qui font qu'une autre année doit passer avant l'édition de 1852-1853 ? C'est que Chauveau est député depuis 1844 ; réélu en 1848 , il est devenu solliciteur général en 1851. II a d'autres préoccupations, sans doute, et peutêtre craint-il un peu «le mépris des hommes affairés "... Quoi qu'il en soit, Charles Guerin est son unique roman, et il faudra attendre presque vingtcinq ans avant qu'il revienne à la littérature (si on laisse de côté ses articles dans le Journal de l'Instruction publique, à partir de 1857).

Une autre indication de $G$. H. Cherrier tend à dévaloriser le sens profond du roman de Chauveau comme si, soupçonnant bien la dimension symbolique de l'œuvre, le courageux éditeur voulait prévenir les coups en désamorçant d'avance l'écriture - et les thèmes de Charles Guérin ${ }^{4}$. Ici encore, il s'agit d'une idée souvent reprise à l'époque, qui aide à mesurer la peur secrète ou inconsciente d'une vérité qui n'échappe pourtant pas tout à fait à la saisie des romanciers, et qui transparaît immédiatement

2. Voir l'exceilente introduction - et les préfaces elles-mêmes - de Guildo Rousseau, Préfaces des romans québécois du xixe siècle, Sherbrooke, Éditions Cosmos, 1970.

3. Dans l'Album littéraire et musical de la Revue canadienne, vol. $1, \mathrm{n}^{\circ} 2$, février 1846 ; vol. II, $n^{\circ} 3$, mars 1847.

4. En 1897, dans son Introduction à la publication en feuilleton de Charles Guérin dans la Revue canadienne (vol. XXXIII, p. 739-741), Ernest Gagnon dira clairement (malgré une prudente parenthèse) un des sens véritables de l'œuvre de Chauveau: "Le problème résultant de la situation de la race conquise (disons cédée pour ne déplaire à personne), en face de la race conquérante, est posé de main de maître dans ce roman dont certaines pages semblent ne dater que d'hier.» 
dans plusieurs textes romanesques du moment. Cherrier précise - et de façon fort lucide cette fois - que l'intrigue amoureuse compte pour presque rien dans le roman qu'il édite. Le "fond" de Charles Guérin, dit-il, ne semblera à plusieurs qu'un prétexte à peinture de mœurs et dissertation politique ou philosophique. C'est qu'en réalité, poursuit-il, les Québécois mènent depuis deux ou trois générations "une vie assez paisible et dénuée d'aventures", et bien que le roman se situe vers 1830 , «époque où les passions politiques et les animosités nationales étaient très vives dans notre pays ", l'auteur n'a trouvé à raconter que des «événements peu saisissants " (comme si, déjà, la dépossession dont sont victimes les Guérin, l'exil du fils aîné et l'épidémie de choléra à Québec n'étaient pas suffisamment dramatiques). Et de s'excuser des paroles extrêmes, des "expressions un peu exagérées" que se permettent quelques-uns des personnages; c'est là le fait, dit-il, de “l'erreur commune du temps". Et enfin, les deux ou trois salauds du roman (lire: Wagnaër, Guillot et Voisin) ne sont là que «parce qu'avec la meilleure volonté du monde, tout ne peut pas être couleur de rose dans un drame ou dans un roman". Tout comme dans l'Histoire, sans doute.

Malgré ces précautions, Charles Guérin révèle quelques-uns des principaux thèmes qui deviendront des clichés dans le roman québécois de la fin $d u x i x^{e}$ siècle, et ces thèmes "inventés" par Chauveau véhiculent, pour la première fois, une bonne part de l'inconscient collectif devant la situation socio-historique "anormale" qui est la nôtre.

La trame d'ensemble de Charles Guérin pourrait se lire ainsi: dépossédé de son patrimoine par l'usurpateur étranger, le héros du roman se refait un destin en travaillant à la réédification nationale; il recommence la première colonisation en mettant sur pied une micro-société à vocation d'abord agricole et patriarcale. La revanche symbolique sur le conquérant s'opère à plusieurs niveaux: refus indigné de la veuve Guérin d'épouser Wagnaër qui convoite le domaine familial; entrée en religion de la fille de l'Anglo-Normand, par dépit et pour ne pas épouser celui auquel son père la destinait; échec probable de Wagnaër dont les affaires périclitent et qui sombre dans l'alcoolisme. Revanche, si l'on peut dire, jusque dans une note de l'auteur, en appendice, où il est démontré que le «God Save the King " était, à l'órigine, un hymne composé au Grand Siècle par JeanBaptiste Lully pour célébrer la gloire de Louis XIV. Et, tout à l'opposé de la dégringolade escomptée de Wagnaër, succès et bonheur sans mélange pour Charles Guérin à la veille de devenir député.

Sur ce patron thématique se greffent quelques idées complémentaires que l'on retrouvera fréquemment aussi dans les cuvres postérieures: difficulté pour le jeune Canadien d'accéder à une carrière libérale où il puisse vraiment se réaliser; fossé entre la nouvelle élite et les "habitants"; univers de la politique considéré comme un repaire d'ambitieux ou un refuge d'incapables; agglomération urbaine vue comme un chaos de bruits et de misères, rempli de travailleurs pauvres et malheureux. Et par-dessus tout, vie rurale et retour à la terre comme voie du salut national. 
Ces grandes lignes du roman sont assorties d'une série d'allusions rapides sur la religion, la "fierté» de certains personnages (qui confine parfois au snobisme); ou bien encore on trouvera, au fil du texte, quelques passages au ton nettement moralisateur qu'on dirait empruntés à Balzac, et qui relèvent du procédé, tout comme l'insertion dans le texte narratif de lettres et de pages de journal intime; de même, au niveau de l'écriture, l'utilisation par Chauveau du parler paysan dans quelques séquences secondaires.

Mais d'abord, qui sont les protagonistes créés par Chauveau? On peut les regrouper en trois familles, autour de liens parentaux ou d'intérêts communs: le clan Guérin, les Wagnaër et consorts, et les Lebrun. À l'ouverture du roman, les Guérin sont au nombre de quatre: la mère, veuve depuis peu, et ses trois enfants: Pierre, dix-neuf ans, Charles, seize ans, et Louise, quinze ans. A qui viennent s'ajouter l'oncle Charlot, assurant l'exploitation de ce qui reste du bien familial, et, plus tard, l'étudiant en médecine Jean Guilbault, ami de Charles. Chez les Wagnaër, il n'y a plus que le père et la fille Clorinde, âgée de dix-sept ans. Trois jeunes Canadiens français gravitent de façon plus ou moins étroite autour du "Jersiais»: François Guillot son commis, et Henri Guillot (dit Voisin), étudiant en droit et autre "ami» de Charles Guérin; et un troisième Guillot, capitaine de navire, survenant de façon inattendue au sixième chapitre de la troisième partie. Et enfin Jacques Lebrun, cultivateur aisé de la campagne montréalaise, et sa fille Marie, ou Marichette; à ce dernier groupe on peut rattacher la mère Paquet, vieille voisine chargée des travaux du ménage, et le père Morelle, voisin chez qui se déroulera la soirée de la mi-carême. Il faut encore mentionner l'avocat Dumont, beau-frère de Jacques Lebrun, chez qui Charles Guérin commence sa cléricature. Quelques autres visages apparaîtront au cours de l'action, mais de façon tout à fait épisodique et jouant plutôt les figurants. Le roman se déroule en quatre parties, de septembre 1830 à l'automne de 1832, l'épilogue nous amenant rapidement en 1837 alors que Guilbault, parti en "excursion de chasse", va se battre dans les échauffourées de Saint-Eustache.

On peut noter deux choses à propos des personnages. D'abord les portraits, à la Balzac, faisant souvent appel à des détails de physiognomonie, en particulier pour Wagnaër: «M. Wagnaër était un homme trapu, surchargé d'embonpoint, son visage était rouge et marqué de petite vérole, et comme frotté d'huile, son nez, plat, ses sourcils, épais et roux, ses yeux, petits et cironnés, ses lèvres, épaisses, sa bouche, très grande et laissant voir deux superbes rangées de dents qui auraient fait honneur à un animal féroce. Avec cette formidable mâchoire, $M$. Wagnaër aurait pu exploiter toute la création. " (p. 23-24) M. Dumont, lui : "Au physique, c'était un petit homme sec, se redressant de son mieux dans sa petite taille, toujours scrupuleusement vêtu de noir, et cravaté de blanc, vif, gai, spirituel, lorsqu'il n'était point tracassé par les plaideurs, très brusque et très maussade parfois, et aussi intelligent que le donnait à croire son large front chauve, ses yeux brillants, son nez aquilin, et tout l'ensemble de son expressive physionomie. » (p. 97) 
Second détail curieux, les trois parents sont veufs: madame Guérin, Wagnaër et Jacques Lebrun. Les deux derniers ont chacun une fille à marier: le parallèle et ce qu'il faut appeler le jeu des fausses pistes, dans la suite du roman, sont aisés à entrevoir. Quant à l'avocat Dumont, il est célibataire. Comme si, dans la vision de Chauveau, l'histoire devait être vécue ici par les fils plutôt que par les pères. Alors que tout réussit aux enfants (Clorinde et le bonheur du cloître, Charles et Marichette en nouveaux seigneurs, Guilbault et Louise en notables, Pierre Guérin en curé adoré de ses ouailles), les parents disparaissent - comme il est normal, au demeurant: madame Guérin est emportée par le choléra, la bonne fortune abandonne Wagnaër qui se remet à boire, ou encore Jacques Lebrun s'efface discrètement dans le récit.

Charles Guérin contient un certain nombre de notations morales ou religieuses qui ne sont pas sans intérêt. Du «spectacle touchant» d'une "naïve dévotion", au début (l'Angélus récité par les enfants Guérin), à la cérémonie mondaine de la prise de voile chez les Ursulines, nous demeurons au niveau de la représentation. Dans le dernier cas au moins, rien ne manque à l'étalage du spectacle religio-mondain: assistance élégante, décor aristocratique de la chapelle, prélude de harpe, entrée solennelle de l'évêque et de son cortège; les spectateurs se pressent et grimpent sur les bancs pour ne rien perdre du déploiement de faste liturgique. Chauveau semble accepter que la religion ne serve qu'aux femmes (madame Guérin assiste à la messe chaque matin): elles y puisent la résignation qui seule adoucit dans leurs âmes sensibles les amertumes de la vie. Pourtant, au retour de Pierre Guérin devenu prêtre, survenant tout juste pour présider à l'enterrement de sa mère, on note que la foi ne vient pas encourager une tristesse stérile; et dans ses prêches de la fin, le curé de la jeune paroisse ne s'attarde pas en longues dissertations sur le dogme: ajustant son enseignement religieux à la vie de labeur rude et austère des nouveaux colons, il parle de justice et de charité plutôt que de pauvreté et de mortification. On soupçonne qu'il doit faire figure de franc-tireur, à une époque où le clergé fonde en général toute doctrine sur un étroit manichéisme et le respect absolu de l'autorité.

Un autre trait observé par Chauveau souligne pertinemment une certaine fierté assez sotte chez un peuple ayant perdu jusqu'à sa petite noblesse ( $y$ en eut-il jamais d'autre ici ?). Madame Guérin, par exemple, éprouve une vive humiliation à retourner vivre dans la vieille maison "qu'elle et son mari avaient quittée quelques années auparavant pour une demeure plus élégante, plus agréable, disons-le aussi, plus prétentieuse »... ( $p .30$ ) L'auteur explique plus loin ce sentiment: "On sait combien les familles riches et distinguées, établies dans les campagnes, se pensent supérieures aux habitants qui les entourent. Le père de Charles n'était point sorti, comme on dit, de la cuisse de Jupiter; cependant la position que l'honnête marchand s'était faite, et l'éducation qu'il avait eue, l'avaient mis en droit de tenir ses voisins à une respectueuse distance. Depuis sa mort, loin de s'affaiblir, l'orgueil de sa famille s'était accru. Madame Guérin avait pour son propre compte, quelques prétentions à la noblesse, et la décadence de sa 
fortune, par une réaction bien légitime, exagérait chez elle le sentiment de sa dignité. Ses enfants, qu'elle ne voulait pas voir complètement déchus, avaient été élevés dans des idées presque aristocratiques." (p. 107) C'est ainsi que Charles considère au début la petite Marichette "comme bien au dessous de lui". De même, à la maison du père Morelle, brave paysan aisé chez qui l'on fête la mi-carême, on voit "une longue table dressée avec un luxe de vaisselle qu'on ne trouve point chez les cultivateurs d'aucun autre pays". Chauveau conclut: "Le père Morelle avait ainsi : salle de réception, salle de danse au besoin et salle à dîner. Que peut-on exiger de plus, même de l'hôte le plus aristocratiquement situé ?" (p.117) Au moment de passer à table, "le vieillard offr[e] galamment la main à la mère Tremblay, avec non moins de grâce qu'en eût déployé en pareille occasion un seigneur à la cour de Louis XIV". (p. 119) Le décor de l'église des Ursulines est à la hauteur des évocations nostalgiques de grandeurs disparues: "son architecture composite (style Louis $X V$ ) toute émaillée de peintures et de dorures, porte un caractère d'élégance aristocratique, qui, pour dater d'un peu loin, ne messied pas au pensionnat le plus à la mode de notre pays". (p. 318) Charles enfin, dans son fief de colonisation, habite "un cottage qui n'est point sans prétention", dans un décor qui reproduit fidèlement celui de la belle maison bâtie jadis pour son père et d'où Wagnaër les a chassés. Ces prétentions accumulées font sourire, mais elles n'en constituent pas moins un élément important du grand processus de compensation psychologique lié au refus de la Conquête.

Chauveau glisse ici et là, dans la trame romanesque, quelques traits de moralisme comme on en trouve souvent chez Balzac: sur le manque d'expérience de la jeunesse: «De quinze à vingt ans on ne sait rien des dégoûtantes vérités de ce monde...» (p. 53); sur la psychologie de l'ambitieux et de l'arriviste, et les complaisances de la société à leur endroit (p. 83-84); sur les dangers de l'excès d'indépendance pour les adolescents, sur la "maladie" du caprice et de la fantaisie (p. 89-94); comparaison entre la douleur des grands et des humbles (p. 257 et suiv.); leçons de l'infortune chez les âmes viles et les âmes d'élite (p. 257). Pourquoi, demande Chauveau, l'enthousiasme pur de la jeunesse s'éteint-il avec l'âge, au contact de la "vie réelle"? pourquoi le monde tolère-t-il si aisément les bassesses, les calculs et les affronts de ceux qui veulent faire leur chemin à tout prix? comment mettre les jeunes gens en garde contre les séductions du rêve et de l'apparence, les arracher aux chimères du romantisme, et leur donner prise sur la réalité? Quant aux idées reçues, elles s'inspirent ici de la morale la plus traditionnelle: les gens ordinaires souffrent autant que les princes, et du malheur les grandes âmes retirent toujours une leçon.

La correspondance et les pages de journal insérées dans la trame du récit constituent un des procédés les plus simples, sinon les plus savants, pour mettre le lecteur au fait de sentiments ou d'événements qui exigeraient par ailleurs de longs détours et des parenthèses risquant de ralentir ou d'interrompre le fil narratif. Ou encore, l'insertion de telles pages peut contribuer à donner à certains épisodes du roman un accent de vérité et d'authenticité plus profond, comme si de tels documents, après tout, ne 
pouvaient être inventés de toutes pièces. La présence des personnages concernés en devient plus convaincante, plus «réelle» (on se souvient qu'Angéline de Montbrun, par exemple, est constituée pour une très large part de lettres échangées, et de pages de journal). Chauveau utilise ce procédé de façon moins étendue: cinq lettres et douze pages environ du journal de Marichette. Sans compter les lettres qui ne sont que mentionnées dans le texte, et dont l'auteur nous fait grâce: pas moins d'une vingtaine de missives de Louise à Charles (et quelques allusions aux brèves réponses de ce dernier), et les cinq ou six brouillons (de Marichette à Charles) que la petite déchire et qu'elle jette au feu; elle fait de même pour une lettre à son amie Émilie, dans laquelle elle racontait ses peines de cœur.

La première de ces lettres, de Pierre Guérin à sa mère, est de loin la plus dramatique: il lui apprend son exil volontaire. Mais la lecture de la mère ne nous apprend rien à nous: suffoquée de douleur, elle s'évanouit dès les premiers mots, avant que nous sachions les détails de cet événement. C'est Charles qui lit à haute voix la lettre d'adieu pour le bénéfice des autres personnages - et le nôtre. La seconde lettre, de Louise à son frère, nous apprend l'amitié qui naît entre Clorinde et elle, et marque le commencement des interrogations de Charles sur un sentiment possible entre lui et la fille de l'Anglais. La troisième, toujours de la sœur au frère, approfondit cette question et parle en outre de Voisin, qui a fait à Wagnaër (devant Louise) un éloge empoisonné de Charles. La quatrième lettre en est une autre dont le message et le sens sont chargés de valeurs négatives. C'est une amie de Marichette qui lui apprend - en toute innocence - les "amours" de Charles et de Clorinde. La dernière lettre enfin est celle du repentir de Charles qui annonce à Marichette sa renonciation à l'héritage de $M$. Dumont pour que la fortune lui revienne entièrement, à elle, en même temps qu'il lui demande pardon pour sa conduite à son égard. Mais la réaction est bien différente de l'effet escompté: «Pour toute réponse, Mlle Lebrun renvoya sous enveloppe la lettre et la renonciation. » (p. 331) Ce renvoi hautain provoque une réconciliation plus rapide - et d'autant plus étonnante: Charles se précipite chez Marichette, et, après deux pages, ils parlent ensemble de mariage.

Le journal de Marichette précède tout juste la lettre d’Émilie (le récit des amours de Charles et de Clorinde): devant le silence de Charles, la petite paysanne se désespère, convaincue qu'il en aime une autre (et elle en reçoit immédiatement la confirmation). En fait, les tristes réflexions de Marichette ne font que décrire la réalité: Charles l'a oubliée peu à peu, ne songeant qu'à Clorinde et à ses chances de devenir le gendre de Wagnaër.

Alors que l'ensemble du roman est écrit en français égal, littéraire, Chauveau traduit les interventions des paysans "aussi textuellement" qu'il le peut, même s'il avoue rapporter leurs conversations en "fragments bien imparfaits". La mère Paquet, les voisins des Lebrun le soir de la mi-carême, chez le père Morelle, le messager qui apporte la lettre d'Émilie à Marichette, les jeunes gens venus assister à la mise aux enchères de la propriété des Guérin ou ceux à qui Charles prêche l'idée d'une nouvelle paroisse: tous 
s'expriment dans un français "meilleur que celui que parlent les paysans des provinces de France où l'on parle le français", précise Chauveau dans une note de la fin (p. 357, note F); ce qui ne l'empêche pas de poursuivre en disant que les colons venus des diverses parties de la France "ont fondu ensemble les particularités de langage et d'accent de leur pays» en un nouveau parler dont la prononciation est néanmoins plus proche de la norme que celle de chacun des parlers respectifs, aux dires de plusieurs voyageurs français. De toute façon, it est étonnant de trouver dans ces transcriptions des traces du langage des paysans de Molière ( «si vous plé "; "J'sommes ben fatigués"; "j'leu-z-en f'rons rabattre un peu»; etc) venu jusqu'à cette époque à travers deux siècles.

Au-delà des thèmes secondaires, Charles Guérin est bien l'histoire du héros qui donne son nom à l'œuvre. Il a seize ans au début, il en aura vingttrois à la fin. Vu sous cet angle, il s'agit véritablement d'un de ces romans d'apprentissage dont le xıxe siècle est prodigue. Un jeune homme quitte l'adolescence et entre dans la vie, perdant une à une ses illusions, s'engageant sur des routes qui s'avèrent des culs-de-sac, trouvant enfin le bonheur après une série de revers, en même temps que sa propre réalisation débouche sur l'engagement patriotique. À l'espèce de paralysie intérieure, qui empêche d'abord Charles Guérin de choisir une voie sûre et définitive dans la conjoncture précise de son époque, succèdent un dynamisme et un esprit d'entreprise qui lui apporteront satisfaction et reconnaissance publique : il est à la veille d'être député, à vingt-trois ans, presque à l'âge même où Chauveau fut élu pour la première fois.

L'organisation de la matière romanesque pourrait être envisagée de la façon suivante: trois séries de questions et de réponses, ou de problèmes et de solutions, en alternances positive et négative. Du côté négatif d'abord, l'antithèse absolue, l'ennemi des Guérin: Wagnaër le fourbe (avec, il est vrai, quelques retournements, alors que madame Guérin finit par éprouver de la sympaihie pour lui; ou encore, au moment ou Wagnaër manifeste un semblant d'amitié à Charles tout en poursuivant, bien sûr, son plan secret); à quoi répondent des groupes de contrastes, de complémentarités ou de balancements, à plusieurs niveaux, et qui participent intimement à la construction du texte. Ensuite, c'est une série d'épisodes ou de situations dans lesquelles se manifestent le désordre, le chaos (dans la nature ou dans les sentiments), et une autre série où la rêverie bâtit un univers ou des états imaginaires dans lesquels l'idéal embellit et ordonne tout. Et enfin, à la description d'une condition socio-historique anormale, faussée, viennent répondre d'une part la conscience de cette situation irrégulière et, après les élans de patriotisme, l'engagement et le travail de reconstruction nationale.

Wagnaër apparaît dans le roman dès les premières pages, mais de façon encore presque anonyme, inoffensive: c'est «un $M$. Wagnaër, étranger venu des îles de la Manche», ou bien "un petit épicier Jersais "; il est tout de suite question d'un autre Anglais, M. Wilby, dont la présence annonçait précisément les façons d'exploiteur de Wagnaër: n'ayant presque rien quand il est arrivé au pays, il a « fait son chemin » grâce à la naïveté de M. Guérin dont il refuse de connaitre les fils désormais, alors qu'il se 
prétendait son grand ami. Chauveau ne tardera pas à montrer en Wagnaër la mine et la contenance "presque sinistres", avec "quelque chose de fatal " dans le regard; son principe absolu est: exploiter de toutes les manières. Voulant s'emparer de la propriété des Guérin, il a tenté d'épouser la veuve, mais celle-ci l'a reçu avec «la plus vive indignation et le mépris le plus écrasant" (on pense à Blanche d'Haberville, dans les Anciens Canadiens). Dans sa lettre d'adieu, Pierre Guérin met sa mère en garde contre ce vampire dont l'unique ambition est d'amener les habitants dans ses filets pour leur extorquer jusqu'à leur dernière chemise (cette image du vampire, de l'araignée et de sa toile ou du monstre dévorant reviendra dans le texte à quelques reprises pour désigner Wagnaër et ses semblables). Dans une lettre à Charles, où elle lui dit les craintes de leur mère devant l'amitié qui l'unit à Clorinde, Louise écrit que ces gens doivent être bien terribles pour que leurs caresses fassent si peur. Une autre lettre de Louise montre à Charles les trois complices (Wagnaër, Guillot et Voisin) comme un trio de sorciers, si laids qu'ils lui font presque peur. Puis, Wagnaër cesse peu à peu d'être l'ennemi juré : même Madame Guérin revient des «préjugés " qu'elle avait à son égard; Charles est invité chez l'Anglais, sa mère est désormais convaincue que "M. Wagnaër a bien changé », et elle envisage avec plaisir la possibilité du mariage de Charles et de Clorinde. D'usurier et de créancier impitoyable qu'il était, Wagnaër devient le grand seigneur magnifique et hospitalier de la fête mémorable de l'été 1831. II n'en poursuit pas moins ses sombres desseins, en entraînant Charles dans une affaire louche de billet endossé qui l'amènera à devoir mettre en vente la propriété familiale, rachetée pour le compte de l'Anglais par un prête-nom. Madame Guérin voit trop tard le complot dont nous apprenons l'explication par l'entremise de l'ami Jean Guilbault, au chapitre 6-bis de la troisième partie du roman, qui nous ramène en arrière dans l'action. Le tout s'achève avec le résumé d'une lettre de Wagnaër à Charles, dans laquelle il le traite tout uniment d'ingrat, parce qu'il fait courir sur lui des bruits infâmants. Mais à l'épilogue, la morale - et la revanche - l'emportent: le sort a tourné, Wagnaër est en mauvaise posture financière et il se remet à boire, au bord de la ruine, tandis que Charles fonde sa paroisse modèle, heureux et admiré de tous.

Une foule de détails, à divers degrés, existent sous forme de contrastes, de complémentarités ou de balancements, comme en équilibre par rapport à ce contraire absolu, à ce pôle négatif qu'est Wagnaër. On constate assez rapidement que la vision de Chauveau a un fort caractère de dualité qui se retrouve aussi bien dans certaine pensée manichéiste que dans de nombreux traits d'écriture classique, montrant toujours, par exemple, un paysage en séries de deux versants, ou bien le calme après la tempête, les traits opposés chez deux personnages, etc.

Le jeu des antithèses commence tout au début, entre les deux frères Guérin: le cadet, Charles, primesautier, irréfléchi peut-être, peu décidé quant au choix d'un état de vie, mais qui songe à la prêtrise; et Pierre, plus mûr, plus calme, ayant longuement pensé à sa vocation, mais qui décide de s'exiler, de partir à l'aventure. C'est pourtant lui qui reviendra de ses 
pérégrinations européennes, ayant été ordonné prêtre à Rome, tandis que Charles, après avoir abandonné l'idée du sacerdoce et l'étude du droit, se fera colon-cultivateur, réalisant ainsi le rêve de jeunesse de son frère. Marichette, pour sa part, a fait des études qui lui ont appris une foule de choses «qui contrast[ent] singulièrement avec sa position» de petite paysanne, et comme telle, on la voit considérée de haut, au début, par Charles, "le Monsieur de la ville". À la soirée de la mi-carême chez le père Morelle, la somptuosité de la table est tout à fait inattendue chez ces gens aux mcurs simples, presque frustes. Parlant de l'éducation, Chauveau ne manque pas de souligner l'autoritarisme qui sévit encore ici alors qu'aux États-Unis la liberté est totale, le choix d'une carrière étant toujours laissé aux jeunes gens. Au bal chez Wagnaër, Charles sera évidemment amené à comparer dans son esprit Clorinde et Marichette, alors que Wagnaër luimême cesse d'apparaître comme le dangereux exploiteur. Par ailleurs Louise Guérin et Clorinde sont les deux reines du bal, mais chacune à sa manière. Lors de la partie d'huittres qui réunit les carabins en goguette, quand Charles a endossé le billet qui le perdra, l'un des convives porte ce toast: « $\grave{A}$ nos billets promissoires et à nos billets doux!», qui convient parfaitement à la situation où se trouve le fils Guérin: n'a-t-il pas mis sa signature au dos du malheureux billet dans l'espoir d'être plus facilement le gendre de Wagnaër? C'est ainsi qu'il perdra tout, songe-t-il, en ayant voulu tout sauver. Il y a un peu de sa faute, du reste, mais sans qu'il l'ait voulu tout à fait: au cours du récit de sa vie, Clorinde raconte à Charles comment elle a promis à sa mère mourante d'épouser le garçon que son père lui choisirait; ce serment a été prononcé sur une petite croix de corail que lui donna alors sa mère. Or, cette croix perdue au bal, c'est Charles lui-même qui la trouve et la rapporte, sans savoir qu'à cause d'elle - et de la promesse dont elle est le signe-il ne pourra jamais épouser Clorinde. II y aurait à souligner ici ce "jeu des fausses pistes», l'évolution du roman laissant croire, en un premier temps, au mariage de Charles et de Clorinde; puis, c'est l'intermède Marichette, suivi d'un retour à Clorinde, de la rupture avec elle et des retrouvailles définitives de Charles et de la petite Marie.

Autres doublets, moins développés ou présentant un intérêt moindre: les deux versions de l'affaire Wagnaër-Guérin, une fois la vente de la propriété accomplie; les traitements à effets contradictoires lors de l'épidémie de choléra; les sens alternés des psaumes récités dans la nuit par Pierre Guérin sur la dépouille de sa mère; le désordre extérieur et l'ordre intérieur (spirituel) lors de la cérémonie des Ursulines.

La nature et le paysage sont aussi vus et décrits en balancements classiques, à la façon de Chateaubriand (à droite... à gauche...; d'un côté... de l'autre...; la tempête... le beau temps...;); ou encore, dans la nuit noire, les lumières de la fête chez les Wagnaër; le calme de la nature, le ciel bleu, et l'âme oppressée de sentiments tristes. Parfois, le désordre de la nature (tempête du début) sera accordé au chaos intérieur et au drame (lettre d'adieu de Pierre).

Le décor, également, est presque toujours équilibré, en deux parties: la maison de madame Guérin, la chambre d'étudiant de Charles à Québec. 
La ville même qui, vue de loin, semble quelque cité magnifique, étagée, monumentale; mais ce n'est là qu'une "éblouissante imposture": elle est en réalité mal construite, pleine de bruits et d'embarras; les toits qu'on aurait dit d'argent sous la lune sont en fer-blanc. Dans l'espèce de "Guérin-ville" de la fin, ou trouvera deux maisons d'égales dimensions, de même apparence, de part et d'autre de l'église: l'école et le presbytère, la science et la foi.

Les bouleversements de la nature sont le plus souvent en harmonie avec une crise intérieure, une situation dramatique ou un état de perturbation morale; ou encore, ils souligneront la présence d'un personnage maléfique, comme les vagues bruyantes et couronnées d'écume, quand Wagnaër va visiter ses nasses; au départ des deux frères Guérin, un vent de tempête souffle pendant huit jours, et c'est le soir de la dernière bourrasque que Charles revient à la maison porteur de la lettre de son frère; à ce moment, du reste, ses vêtements sont dans un désordre complet, lui qui d'habitude soigne tellement sa mise; et c'est à la lecture des premiers mots de cette lettre d'adieu que madame Guérin sombre dans une crise nerveuse. En même temps qu'on lit ce message tragique, où Pierre écrit qu'il est préférable qu'on le croie mort désormais, le gros arbre près de la maison est frappé par la foudre: "l'orme de la famille qui tombe ! "s'écrie Louise.

À la soirée du père Morelle, la vieille déguisée en mi-carême. affublée d'oripeaux et de guenilles, le visage noirci, l'air d'une sorcière, met Charles en garde contre un danger qui le guette: au retour, la voiture est renversée et Marichette et lui se retrouvent en mauvaise posture, au bord d'une coulée. Dans le vacarme et le chahut de la partie d'huîtres, l'imagination et les nerfs de Charles sont ébranlés. Quand Wagnaër lui suggère de mettre la propriété en vente, il est encore tout étourdi, et au moment des enchères, il est pris de vertige: l'église et les habitants semble tourner autour de lui et il a l'impression que le sol se dérobe sous ses pas. Le spectacle de la ville, à Québec, crée encore une impression de désordre et de grouillement cahotique: Louise croit qu'elle en perdra la tête; l'épidémie de choléra qui s'abat sur la région constitue le sommet de la confusion; madame Guérin est atteinte et meurt rapidement; Chauveau décrit les convois funèbres tumultueux, dans la nuit, et pendant sa veille, Pierre est pris d'une syncope dont il sort pour trouver dans les psaumes qu'il lit la colère de Dieu qui le plonge dans la confusion et la terreur. Le lendemain, il sombre dans une fièvre violente qui fait craindre pour sa raison.

Tout à l'opposé, l'imagination veut faire échec à la désorganisation et aux bouleversements de la réalité, même si elle est parfois considérée comme stérile, ennemie de l'action. Le dernier jour des dernières vacances nous montre Pierre et Charles en longues rêveries sur le paysage familier qu'ils vont quitter; à Québec, Charles passe de nombreux moments à suivre de séduisants fantômes, inspiré par la lecture des Martyrs de Chateaubriand. Avec ses camarades Guilbault et Voisin, il échafaude des plans d'action politique extravagants et inoffensifs. Plus tard, madame Guérin rêve à l'avenir de Charles qu'elle envisage rempli de réalisations heureuses. Au moment de la vente de la propriété, les «gens positifs"se 
demandent pourquoi Charles pose à la victime, crie à l'intrigue et à l'imposture: il n'est après tout qu'un poète, un visionnaire...

Mais c'est un poète qui, à l'image de plusieurs de ses contemporains sans doute, a vécu dans les revers et l'indécision même de son propre destin l'inquiétude et l'inconfort absolu d'une société anormale, d'une histoire tronquée. Le doute l'assiège dès le moment de choisir une carrière: pour le jeune Québécois d'alors, quatre voies seulement sont ouvertes; il doit être prêtre, médecin, notaire ou avocat. Impossible d'entrer encore au gouvernement; le commerce est aux mains des Anglais; et la hardiesse lui manque pour créer des industries nouvelles. Pierre Guérin s'exilera, incapable d'affronter seul la "fatalité" qui paralyse les énergies. La véritable raison de cette situation troublante, elle est dans la question posée par Voisin: "est-ce que nous avons un pays, nous autres? Vous parlez sans cesse de votre pays: je voudrais bien savoir si le Canada est un pays pour quelqu'un?" (p. 54) (Cette dé-paysation est symbolisée ici par la dé-possession dont Charles est victime, bien entendu.) Certains prônent l'anglicisation, d'autres l'américanisation, puisqu'aussi bien, dès lors, presque tous les Québécois parlent l'anglais: "comme il faut" d'abord, et même les ouvriers (non sans quelque réticence, il est vrai: "Mon Dieu, que c'est tannant d'être toujours obligée de parler anglais!"s'écrie l'épouse de l'aubergiste du faubourg Saint-Jean). Pour ce qui est de la loi, nous avons tous les codes imaginables: droit romain, vieux droit français, nouveau droit français, droit anglais, "ce qui fait que nous n'en avons pas du tout". (p. 62) En définitive, c'est Pierre Guérin, de retour d'Europe, qui pose le problème fondamental: en France, on l'a cru Anglais, Italien, Français de province... «J'ai voulu dire que j'étais Canadien français. Autant aurait-il valu leur annoncer que je venais de la lune. [...] Ceux qui me comprirent crurent que j'étais un sauvage, et on m'accabla de mille sottes questions. " (p. 311)

La réponse globale à cette absurdité de l'histoire n'est certes pas dans la fuite à la façon de Pierre Guérin; non plus que dans l'hésitation, comme pour Charles; $\mathrm{nl}$, sans doute, dans l'intrigue et l'arrivisme personnel dans lequel s'engage Voisin. Le labeur silencieux, courageux et obstiné de Guilbault? C'est là déjà une voie de salut. L'aîné des frères Guérin avait entrevu la solution: "L'énergie de toute une population bien employée et constamment employée finirait par user à la longue la chaîne du despotisme colonial." Mais il faut des meneurs, des chefs: Charles Guérin deviendra l'un de ceux-là, avec son ami Jean Guilbault dont le thème de prédilection - il fallait s'y attendre - est celui de la revanche providentielle des berceaux: "Pensez-vous qu'une nationalité aussi vivace se détruise dans un jour?" (p. 65) II faut donc encourager la natalité, se priver de luxe et de superflu, créer et développer l'industrie locale. C'est à quoi Charles et lui s'aṕpliqueront à la fin. En s'efforçant de persuader leurs jeunes compatriotes de ne pas s'exiler en Nouvelle-Angleterre, vers les nouvelles filatures et les salaires «intéressants», en formant une petite société pour le développement des terres incultes depuis les quatre cents arpents hérités de M. Dumont, ils fondent leur doctrine en trois points: lâcheté 
d'abandonner son pays, danger de perdre sa foi et ses mœurs à l'étranger, avantage et patriotisme de fonder de nouveaux établissements dans notre propre pays. C'est, en gros, tout le programme de Maria Chapdelaine et des innombrables récits de "l'heure des vaches" dans le roman québécois.

Mais il s'agit là, en réalité, d'un perpétuel recommencement (un peu comme Samuel Chapdelaine, qui pousse toujours plus au nord): le texte lui-même suggère assez que ce nouvel enracinement reproduit exactement l'installation des colons au $\mathrm{XVII}^{\mathrm{e}}$ siècle: la première année est consacrée à l'arpentage des terres et à l'ouverture du chemin, par corvées, "comme cela se pratiquait dans les premiers temps du pays" (p. 340); puis vient le défrichement et l'aménagement du sol, supervisés par Charles lui-même: et la construction d'une chapelle en bois (qui sera remplacée par une église de pierre, peu après); ensuite l'école, et le moulin à scie. De façon significative, les constructions qui entourent l'église s'appellent «le Fort», "par un reste de tradition militaire qui remonte aux premiers temps de la colonie». (p. 343) Comme si rien n'avalt eu lieu, on refait l'implantation. Aux dernières pages, Guilbault prend-il part à la rébellion de 1837, dans la bagarre de Saint-Eustache, qu'il reconnaît ensuite «la folie de cette expédition ».

Le salut par la terre, la vocation agricole? Ce serait ramener le roman de Chauveau à des limites trop étroites. D'ailleurs, comme son créateur lui-même, et malgré ses réticences, Charles Guérin sera député. L'engagement politique, la lutte constitutionnelle avec tous ses avatars, était sans doute la voie de résistance la plus raisonnable, malgré même les piétinements et les tergiversations. "Se faire un pays" dans ces conditions serait une victoire à l'usure.

Malgré ses maladresses, Charles Guérin demeure la première œuvre valable de la littérature québécoise. Au milieu du xixe siècle, un jeune écrivain disait enfin, par le biais d'une fiction romanesque transparente. quelques-unes des conséquences les plus lourdes et les plus dramatiques de la Conquête: cul-de-sac des professıons classiques déjà encombrées: paralysie du dynamisme et des énergies vitales; exploitation systématique des ressources importantes aux mains des seuls Anglais. L'avenır et les possibilités de développement normal de toute une nation anéantis pour presque un siècle encore. Si l'on y réfléchit, la seule voie possible était au fond celle que trace Charles Guérin: l'enracinement dans le sol national, qui sera l'essentiel de tout le prêche patriotique jusqu'à l'entre-deuxguerres. C'est le sursaut habituel des peuples conquis, de s'agripper farouchement à leur terre; sinon ils se condamnent à la dispersion et à l'errance perpétuelle. Mais c'était là le salut et la perte, l'assurance de durer et l'enlisement progressif. De toute façon, comment en aurait-il été autrement?

Le refus inconscient de la Conquête et les revanches symbolıques qui se retrouveront dans la plupart des romans de la fin du siècle sont déjà exprimés dans l'œuvre de Chauveau, à cette différence près que la mise en forme de Charles Guérin constitue une véritable structure romanesque, sans démonstration idéologique et sans thèse. Le modèle qui ressort de la lecture proposée ici est fort simple: d'un côté l'ennemi, le conquérant, 
l'Anglais; les désordres de la nature et les perturbations morales, intérieures; l'état socio-historique anormal. De l'autre: les jeux de contrastes, de complémentarités, d'antithèses, qui construisent le roman; l'ordonnance idéale rebâtie dans l'imaginaire et le rêve; et la solution de l'engagement patriotique dans la réédification nationale, le retour à la terre. Voilà, schématisé à l'extrême, un des plans de déchiffrement possibles de "notre troisième roman".

Jean-Pierre Duquette Université McGill 